\title{
Mitteilungen aus der Gesellschaft für afrikanisches Recht (2014)
}

\author{
Harald Sippel ${ }^{1}$
}

In vergangenen Ausgaben der Zeitschrift „Recht in Afrika“, aber auch im „Jahrbuch für afrikanisches Recht" und an anderer Stelle, wurde bereits häufig über die Aktivitäten der Gesellschaft für afrikanisches Recht e.V. berichtet, um deren Mitglieder und die interessierte Öffentlichkeit vor allem über die stattgefundenen Tagungen zu informieren und auf die geplanten Veranstaltungen unserer wissenschaftlichen Vereinigung hinzuweisen. Hierfür haben sich die „Mitteilungen aus der Gesellschaft für afrikanisches Recht“ als probates Mittel zur Information des avisierten Adressatenkreises erwiesen. ${ }^{2}$

Im Weiteren legen zahlreiche Berichte zu den Jahrestagungen ${ }^{3}$ und zu den Sondertagungen ${ }^{4}$ der Gesellschaft für afrikanisches Recht eindrücklich Zeugnis über die Fülle und den Erfolg der ausgerichteten Veranstaltungen ab. Neben diesen Tagungsberichten sind die „Mitteilungen aus der Gesellschaft für afrikanisches Recht“ nicht nur zu Informationszwecken geeignet, sondern sie vermögen auch als institutionelles Gedächtnis für die Aktivitäten unseres Vereins zu dienen, um in unserer schnelllebigen und vergesslichen Zeit diese der Nachwelt zu vergegenwärtigen und zu bewahren. Aus diesen Gründen soll auch in der Online-Ausgabe unserer Zeitschrift wieder regelmäßig über die vielfältigen Tätigkeiten der Gesellschaft für afrikanisches Recht berichtet werden.

1 Privatdozent Dr. Harald Sippel ist Vorsitzender des Vorstandes der Gesellschaft für afrikanisches Recht e.V. (E-Mail: harald.sippel@rechtinafrika.de).

2 Siehe Recht in Afrika 1 (1998), S. 135 f. und S. 271 f.; Recht in Afrika 2 (1999), S. 117 und S. 255 f.; Recht in Afrika 3 (2000), S. 105 f. und S. 240 f.; Recht in Afrika 4 (2001), S. 103 und S. 235 f.; Recht in Afrika 5 (2002), S. 109 f. und S. 259 f.; Recht in Afrika 6 (2003), S. 107 und S. 247; Recht in Afrika 7 (2004), S. 119 f. und S. 249; und Recht in Afrika 13 (2010), S. 291 f.

3 Über die Jahrestagungen der Gesellschaft für afrikanisches Recht informieren zahlreiche Tagungsberichte, siehe beispielsweise Madlener, Kurt, Überblick über die Jahrestagungen 1975-1979 der Gesellschaft für afrikanisches Recht, in: Jahrbuch für afrikanisches Recht 1 (1980), S. 155-157; Madlener, Kurt, Überblick über die Jahrestagungen 1980-1984 der Gesellschaft für afrikanisches Recht, in: Jahrbuch für afrikanisches Recht 5 (1984), S. 203-205; Weingärtner, Dieter, Jahrestagung der Gesellschaft für afrikanisches Recht 1985 (Heidelberg, 8./9. November 1985), in: Jahrbuch für afrikanisches Recht 6 (1985/86), S. 283-286; Grozinger, Dieter, XIIe Conférence annuelle de l'Association de droit africain (Marburg, 14 et 15 novembre 1986), in: Jahrbuch für afrikanisches Recht 6 (1985/86), S. 325-328; Spellenberg, Ulrich, 12.-19. Jahrestagungen der Gesellschaft für afrikanisches Recht (Heidelberg, 14./15. November 1986, 6./7. November 1987, 4./5. November 1988, 10./11. November 1989, 9./10. November 1990, 8./9. November 1991, 6./7. November 1992, 5./6. November 1993), in: Jahrbuch für afrikanisches Recht 7 (1993), S. 197-199; Sippel, Harald, Bericht über die XVIII. Jahrestagung der Gesellschaft für Afrikanisches Recht e.V. in Heidelberg am 6. und 7. November 1992, in: Verfassung und Recht in Übersee 26 (1993), S. 212-216; Sippel, Harald, Bericht über die XIX. Jahrestagung der Gesellschaft für Afrikanisches Recht e.V. 


\section{Publikationen}

Im Rüdiger Köppe Verlag in Köln erschien von 1998 bis 2013 mit 16. Jahrgängen unsere Zeitschrift „Recht in Afrika“ in gedruckter Form. Aus wirtschaftlichen Gründen sah sich der Verlag veranlasst, den Herausgebervertrag mit den bisherigen Konditionen mit Wir-

in Heidelberg am 5. und 6. November 1993, in: Verfassung und Recht in Übersee 27 (1994), S. 358-364; Spellenberg, Ulrich/Harald Sippel, 20. Jahrestagung der Gesellschaft für afrikanisches Recht (Heidelberg, 4./5. November 1994), in: Jahrbuch für afrikanisches Recht 8 (1980), S. 175-180; Spellenberg, Ulrich/Harald Sippel, Bericht über die XX. Jahrestagung der Gesellschaft für Afrikanisches Recht e.V. in Heidelberg am 4. und 5. November 1994, in: Verfassung und Recht in Übersee 28 (1995), S. 386-392; Woodman, Gordon R., $21^{\text {st }}$ Annual Meeting of the African Law Association (Heidelberg, November 3 to 4, 1995), in: Jahrbuch für afrikanisches Recht 9 (1995), S. 199-201; Reiher, Hannes/Laura Alester, Administration of Justice in Africa - Effectiveness, Acceptance and Assistance, Report on the Joint Conference of the Protestant Academy Loccum and of the African Law Association (Rehburg-Loccum, Germany, 30 November to 2 December 2007), in: Recht in Afrika 10 (2007), S. 265-268; Lohse, Volker, Comparing Law in Africa - 35. Jahrestagung der Gesellschaft für afrikanisches Recht vom 6.-8. November 2009 in Würzburg, in: Recht in Afrika 13 (2010), S. 285-289; Nixdorf, Lisa, Formal/Informal Law and Economic Development in Africa. Report on the Joint Conference of the Institute of African Studies at the University of Leipzig and of the African Law Association in Leipzig, Germany, in: Recht in Afrika 14 (2011), S. 133-136; Diehl, Eva/Kimon Haars, Report on the Conference "Legal Transformation in North Africa and Decentralization in Africa" in Heidelberg, 4-5 November 2011, in: Recht in Afrika 15 (2012), S. 155-162; Himmer, Thomas/Robert Owino, Bericht zur Jahrestagung der Gesellschaft für afrikanisches Recht e.V. (African Law Association), Humboldt-Universität zu Berlin, 9.-10.11.2012, in: Recht in Afrika 16 (2013), S. 113-117.

Einige Hefte der Zeitschrift "Recht in Afrika" erschienen zugleich als Konferenzausgaben der Jahrestagungen der Gesellschaft für afrikanisches Recht, siehe beispielsweise Meinecke, Oliver/Harald Sippel (eds.), Administration of Justice in Africa - Effectiveness, Acceptance and Assistance, $33^{\text {rd }}$ Annual Conference of the African Law Association, Loccum, Germany, November $30^{\text {th }}-$ December $2^{\text {nd }}, 2007$, Köln, Rüdiger Köppe Verlag, 2008, zugleich Heft 1 von Recht in Afrika 11 (2008); Marauhn, Thilo/Oliver Meinecke (eds.), Regional Integration in Africa, $34^{\text {th }}$ Annual Conference of the African Law Association, Giessen, Germany, $7^{\text {th }}-9^{\text {th }}$ November, 2008, Köln, Rüdiger Köppe Verlag, 2009, zugleich Heft 2 von Recht in Afrika 12 (2009); Elliesie, Hatem/Chadidscha Schoepffer/Thilo Marauhn (eds.), Formal/Informal Law and Economic Development in Africa, $36^{\text {th }}$ Annual Conference of the African Law Association, Leipzig, Germany, $5^{\text {th }}-6^{\text {th }}$ November 2010 , Köln, Rüdiger Köppe Verlag, 2012, zugleich Heft 1 von Recht in Afrika 15 (2012).

4 Siehe zu den Sondertagungen der Gesellschaft für afrikanisches Recht beispielsweise die Tagungsberichte von Beke, Dirk, The Training of African Jurists (Speyer, 15 and 16 April 1985), in: Jahrbuch für afrikanisches Recht 6 (1985/86), S. 257-260; Brink, Josef, Die Ausbildung afrikanischer Juristen (Speyer, 15. und 16. April 1985), in: Jahrbuch für afrikanisches Recht 6 (1985/86), S. 261-268; Dogbevi, Agbenyo Komikuma, La formation des juristes africains (Spire, 15 et 16 avril 1985), in: Jahrbuch für afrikanisches Recht 6 (1985/86), S. 269-271; Keller, Marita, Internationale Wirtschaftsbeziehungen afrikanischer Staaten (München, 4./5. Juni 1986), in: Jahrbuch für afrikanisches Recht 6 (1985/86), S. 313-316.

Siehe auch die Tagungsbände: Bryde, Brun-Otto/Lothar Gündling/Gabriele Oestreich (Hrsg.), Zwischen Collective Self-Reliance und Entwicklungshilfe, Internationale Wirtschaftsprobleme afrikanischer Staaten, Marburg, 1989, und African Law Association (ed.), The African Charter on Human and Peoples' Rights: Development, Context, Significance; Papers of a Symposium of the African Law Association held in Maastricht in 1987, Marburg 1991. 
kung zum 31. Dezember 2013 zu kündigen. Um weiterhin die Herausgabe einer möglichst kostengünstigen Zeitschrift zu ermöglichen, fasste der Vorstand ${ }^{5}$ der Gesellschaft für afrikanisches Recht im Januar 2014 den Beschluss, dass die Zeitschrift künftig bei der Nomos Verlagsgesellschaft in Baden-Baden in elektronischer Form als Open-Access-Publikation fortgeführt werden soll; ein Vorhaben, dass nach einigem Aufwand relativ zeitnah realisiert werden konnte. Ein zusätzlicher Vorteil der Online-Veröffentlichung besteht darin, dass unsere Zeitschrift nunmehr weltweit, also vor allem auch in afrikanischen Staaten, kostenfrei und ohne Zugangsbeschränkung gelesen werden kann. Wir hegen die berechtigte Hoffnung, dass unsere Zeitschrift und gleichzeitig unsere Gesellschaft durch diesen Schritt in der Öffentlichkeit verstärkt wahrgenommen werden wird. Unser Dank gilt vor allem den Mitgliedern der Gesellschaft für afrikanisches Recht, die mit ihrem Jahresmitgliedsbeitrag die Herausgabe der Zeitschrift ermöglichen. Damit wird nicht nur ein wichtiger Beitrag zur Erforschung und Entwicklung des Rechts in Afrika geleistet, sondern es wird auch Wissenschaftlerinnen und Wissenschaftlern, die sich mit Recht in Afrika befassen, insbesondere afrikanischen Kolleginnen und Kollegen, ein bedeutendes Publikationsforum zur Verfügung gestellt.

Mit dem vorliegenden Heft von „Recht in Afrika“ ist bereits der 17. Jahrgang unserer Zeitschrift erschienen. Auch in ihrem Online-Format verfolgt „Recht in Afrika“ das Ziel, ein Informations- und Diskussionsforum für die unterschiedlichen und vielfältigen Ausprägungen von Recht, das auf dem afrikanischen Kontinent zur Anwendung kommt, zu sein.

Unsere wissenschaftliche Vereinigung gibt im Rüdiger Köppe Verlag in Köln außerdem die „Schriftenreihe der Gesellschaft für afrikanisches Recht“" heraus, welche nunmehr bereits vier Bände umfasst. ${ }^{6}$

\section{Homepage}

Informationen über die Tätigkeiten und Veranstaltungen der Gesellschaft für afrikanisches Recht sind im Internet unter www.rechtinafrika.de abrufbar.

5 Mitglieder des Vorstandes der Gesellschaft für afrikanisches Recht e.V. sind gegenwärtig: Eva Diehl M.A., Dipl.-Jur. Hatem Elliesie, Kimon Haars, RA Prof. Dr. Hartmut Hamann, Prof. Dr. Thilo Marauhn, Dr. Oliver Meinecke, Prof. Dr. Wilhelm J.G. Möhlig, RA Dr. Dirk Otto, Prof. Dr. Oliver Ruppel, Dr. Katrin Seidel, PD Dr. Harald Sippel (Vorsitz) und Prof. Dr. Ulrike Wanitzek.

6 Band 1: Böhler, Katja, Die Landfrage in Simbabwe. Eine zeitgeschichtlich-juristische Untersuchung, Köln, 2006; Band 2: Ebeku, Kaniye S.A., Oil and the Niger Delta People in International Law. Resource Rights, Environmental and Equity Issues, Köln, 2006; Band 3: Grütjen, Klaus, Lokale Selbstverwaltung im Spannungsfeld von afrikanischer Tradition und europäischer Moderne. Dezentralisierung und Dekonzentration in den frankophonen Staaten Westafrikas am Beispiel Burkina Fasos, Köln, 2011; Band 4: Seidel, Katrin, Rechtspluralismus in Äthiopien. Interdependenzen zwischen islamischem Recht und staatlichem Recht, Köln, 2013. 


\section{Kooperation}

Über das Austauschprogramm für berufserfahrene junge Juristen aus Burundi, der Demokratischen Republik Kongo und Ruanda mit der Justiz in Baden-Württemberg, das von der Robert Bosch Stiftung (Stuttgart, Deutschland) gefördert und von der Gesellschaft für afrikanisches Recht e.V. als antragstellende Institution begleitet wird, wurde in dieser Zeitschrift bereits informiert. ${ }^{7}$ Hierzu liegt nun auch ein Bericht eines Teilnehmers des Austauschprogramms vor. ${ }^{8}$

Im Kalenderjahr 2014 führte eine weitere Kooperation der Gesellschaft für afrikanisches Recht mit der Willy Brandt School of Public Policy an der Universität Erfurt zu der nachfolgend näher vorgestellten Tagung.

\section{Jahrestagung 2014}

Die 41. Jahrestagung der Gesellschaft für afrikanisches Recht fand als Kooperationstagung gemeinsam mit der Willy Brandt School of Public Policy an der Universität Erfurt, Deutschland, auf dem Campus und in den historischen Räumlichkeiten der KatholischTheologischen Fakultät auf dem Erfurter Domberg vom 7. bis 8. November 2014 statt.

Zum Tagungsthema Law and Development in Africa: Between Empowerment and Conditionality hielten zunächst der Verfasser dieses Textes und sodann Prof. Dr. Florian Hoffmann (Willy Brandt School of Public Policy) einführende Referate (Foundations of ,Law and Development " und The Multiple Reincarnations of the ,Law and Development "Movement).

Im von Laura Held (Willy Brandt School of Public Policy) moderierten zweiten Tagungsabschnitt präsentierten Edward Silvestre Kaweesi (Willy Brandt School of Public Policy) zum Thema Human Security versus State Capacity in Contemporary Rwanda, Dr. Katrin Seidel (Max-Planck-Institut für Ethnologie, Halle an der Saale, Deutschland) zu State Formation through Constitution-making: South Sudan's Constitutional Genesis in the Light of Rule of Law Frameworks und Dr. Jude Kagoro (Universität Bremen, Deutschland) über Law, Policing and Society Paradox, ein Vortrag, in dem er die polizeilichen Aktivitäten zur Durchsetzung von Recht und Ordnung in Uganda kritisch analysierte.

Im dritten Tagungsabschnitt unter Leitung von Prof. Dr. Florian Hoffmann sprachen Prof. Dr. Frederick Jjuuko (Makerere Universität, Uganda) über Law, Gender, and Development und Dr. Annamarie Bindenagel Šehovič (Universität Erfurt, Deutschland) zu Legal Regulation of HIV/AIDS in ausgewählten afrikanischen Staaten.

7 Siehe den Bericht von Sippel, Harald, Förderung der Justiz in Afrika: Austauschprogramm zwischen Baden-Württemberg und zentralafrikanischen Staaten, in: Recht in Afrika 17 (2014), S. 113-115.

8 Siehe den Bericht von Balinge Kahombo, Summary Report on the 'Exchange Programme between Young Lawyers from Burundi, Rwanda and the Democratic Republic of Congo and the Judiciary of Baden-Württemberg (Germany)' from 5 to 18 October 2014, in diesem Heft. 
Dr. Oliver Meinecke (Bundesministerium für wirtschaftliche Zusammenarbeit und Entwicklung, Deutschland) moderierte den vierten und letzten Tagungsabschnitt, in dem Wilfried Zoungrana (Willy Brandt School of Public Policy) zu The (Legal) Linkages of (Anti-) Terrorism and Development Discourse und Prosper Simbarashe Maguchu (Justus-LiebigUniversität Gießen, Deutschland) über Affirmative Action: Black Empowerment Law and its Effect on Economic Development in Zimbabwe and South Africa vortrugen.

Unser Dank gilt den Rednerinnen und Rednern sowie allen Besuchern, welche die Diskussionen durch ihre Wortbeiträge bereichert haben. Besonders dankbar sind wir dem Direktor und der Koordinatorin der Willy Brandt School of Public Policy an der Universität Erfurt, Herrn Prof. Dr. Florian Hoffmann und Frau Laura Held, sowie ihren Mitarbeiterinnen und Mitarbeitern für die hervorragende Organisation der Kooperationstagung.

\section{Jahrestagung 2015}

Die 42. Jahrestagung der Gesellschaft für afrikanisches Recht wird als Kooperationstagung mit der Hochschule Harz in Wernigerode, Deutschland, zum Thema "Rechtstransfer in Afrika" (Arbeitstitel) am 6. und 7. November 2015 stattfinden. Nähere Informationen werden zu gegebener Zeit den Mitgliedern der Gesellschaft und auf der Homepage unserer Gesellschaft mitgeteilt. 


\section{Impressum}

Recht in Afrika - Law in Africa - Droit en Afrique - Zeitschrift der Gesellschaft für afrikanisches Recht

Herausgeber: Gesellschaft für afrikanisches Recht e.V., vertreten durch den Vorsitzenden des Vorstandes, Herrn Dr. Harald Sippel, Barbarossastraße 31, 09112 Chemnitz

Schriftleitung: RA Prof. Dr. Hartmut Hamann, Falkertstraße 82, D-70193 Stuttgart Telefon: +49 (o) 711 120950-30 | Fax : +49 (0) 711 120950-50

Mail: hamann@hamann-legal.de

Erscheinungsweise: 4 Ausgaben pro Jahr

Druck und Verlag: Nomos Verlagsgesellschaft mbH \& Co. KG - Waldseestr. 3-5 D-76530 Baden-Baden. Telefon +49-7221-2104-0 / Fax 49-7221-2104-27

E-Mail:nomos@nomos.de

Anzeigen: sales friendly Verlagsdienstleistungen - Inh. Frau Bettina Roos - Pfaffenweg 15 - D-53227 Bonn Telefon +49-228-97898-o / Fax +49-228-97898-20 E-Mail:roos@sales-friendly.de

Urheber- und Verlagsrechte: Die Zeitschrift und alle in ihr enthaltenen einzelnen Beiträge sind urheberrechtlich geschützt. Jede Verwertung außerhalb der engen Grenzen des Urheberrechtsgesetzes ist ohne Zustimmung des Verlages unzulässig. Das gilt insbesondere für Vervielfältigungen, Übersetzungen, Mikroverfilmungen und die Einspeicherung und Verarbeitung in elektronischen Systemen. Der Nomos Verlag beachtet die Regeln des Börsenvereins des Deutschen Buchhandels e.V. zur Verwendung von Buchrezensionen.

ISSN 2363-6270

\section{Nomos}

